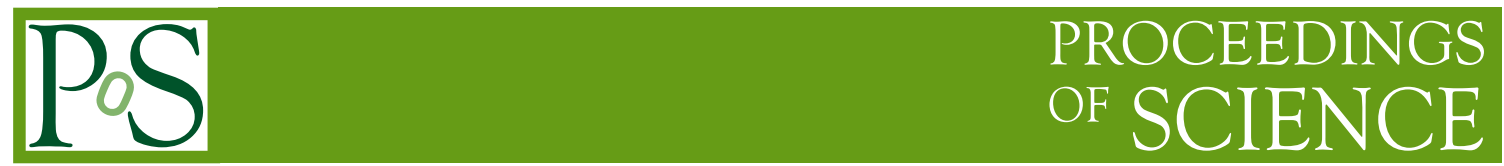

\title{
Energy dependence of hadron production in EPOS
}

\section{Klaus WERNER*}

SUBATECH, Université de Nantes - IN2P3/CNRS-EMN, Nantes, France

E-mail: werner@subatech.in2p3.fr

One may divide the collision zone in high energy nuclear collisions into a central part ("core") with expected high energy densities, and a peripheral part ("corona") with smaller energy densities more like in pp or pA collisions. We discuss the consequences of the core-corona separation within the EPOS approach, by investigating particle production in the energy range 20-200 AGeV.

The 3rd edition of the InternationalÂăWorkshop - The Critical Point and Onset of Deconfinement July 3-7 2006

Galileo Galilei Institute, Florence, Italy

${ }^{*}$ Speaker. 
In high energy nuclear collisions, the peripheral nucleons of either nucleus essentially perform independent $\mathrm{pp}$ or $\mathrm{pA}$-like interactions with a very different particle production compared to the high density central part. For certain observables, this "background" contribution completely spoils the "signal" and to properly interpret data we need to subtract this background.

In order to get quantitative results, we need a simulation tool, and here we take EPOS [1], which has proven to work very well for pp and dAu collisions at SPS and RHIC. EPOS is a parton model, so in case of a heavy ion collision there are many binary interactions creating partons, which then hadronize by employing a phenomenological procedure called string fragmentation. Here, we modify the procedure: we have a look at the situation at an early proper time $\tau_{0}$ long before the hadrons are formed: we distinguish between string segments in dense areas (more than $\rho_{0}$ segments per unit proper volume) from those in low density areas. In the following, we will use $\tau_{0}=1$ and $\rho_{0}=1 \mathrm{fm}^{-3}$. We refer to high density areas as core and to low density areas as corona.

In figure 1, we show two randomly chosen examples of semi-peripheral (40-50\%) AuAu collisions at $200 \mathrm{AGeV}$ (cms), simulated with EPOS. We observe large fluctuations event by event, simply reflecting the randomness of the positions of binary nucleon-nucleon collisions. There is an important contribution from the low density area, contributing roughly $20 \%$ to the final particle production. But much more importantly, the relative weight of this contribution depends strongly on particle type and transverse momentum. For central collisions, the low density contribution is obviously less important, for more peripheral collisions this contribution will even dominate.

How do these low density contributions interact with the expanding core? Even a system of noninteracting particles expands with the velocity of light (reflecting the outward moving particles). Inward moving particles may be absorbed by the core, on the other hand the core edges start to hadronize at the same time with a good chance that early hadronization and absorption compensate each other. So we ignore any interaction for the moment. But even if part of the low density contribution will be absorbed, there will be a sizable effect.

In order to make a quantitative statement, we adopt the following strategy: the low density part will be treated using the usual EPOS particle production which has proven to be very successful in pp and dAu scattering [1] (the peripheral interactions are essentially pp or pA scatterings). For the high density part, we simply try to parameterize particle production in the most simple way possible. It is not at all our aim to provide a microscopic description of this part.

In practice, we first divide the EPOS string segments into core and corona contribution, as discussed earlier. We then consider the core contributions more closely, in transverse slices characterized by some range in $\eta=0.5 \ln (t+z) /(t-z)$. Since string segments show a Bjorken-fluid-like behavior, the particles in a segments around $\eta$ move with rapidities close to $\eta$. Connected core regions in a given segment are considered to be clusters, whose energy and flavor content are completely determined by the corresponding string segments. Clusters are then considered to be collectively expanding: Bjorken-like in longitudinal direction, with in addition some transverse expansion. We assume that the clusters freeze out at some given energy density $\varepsilon_{\text {hadr }}$, having acquired at that moment a collective radial flow with a linear radial rapidity profile from inside to outside characterized by the maximal radial rapidity $y_{\text {rad }}$. In addition, we impose an azimuthal asymmetry being proportional to the the initial spacial eccentricity $\varepsilon$, where the coefficient of proportionality $f_{\text {ecc }}$ is a parameter. By imposing radial flow, we have to rescale the cluster mass correspondingly in order to conserve energy. Hadronization then occurs according to covariant phase space. The 

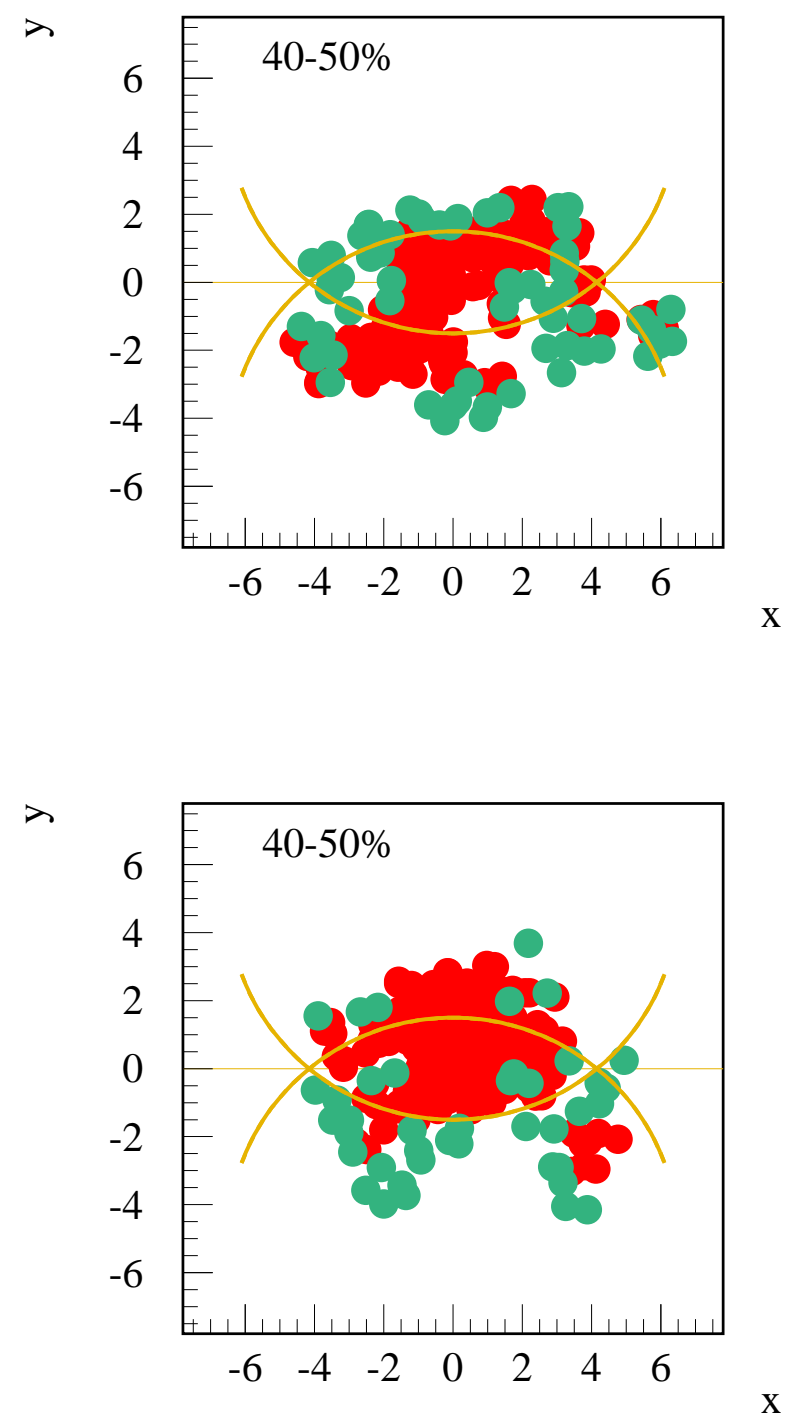

Figure 1: Two Monte Carlo realizations of semi-peripheral (40-50\%) AuAu collisions at $200 \mathrm{GeV}$ (cms). We show in red string segments in high density areas (core), and in green the string segments in low density environments (corona). The circles are put in just to guide the eye: they represent the two nuclei in hard sphere approximation. We consider a projection of segments within $z= \pm 0.8 \mathrm{fm}$ to the transverse plane $(\mathrm{x}, \mathrm{y})$. 


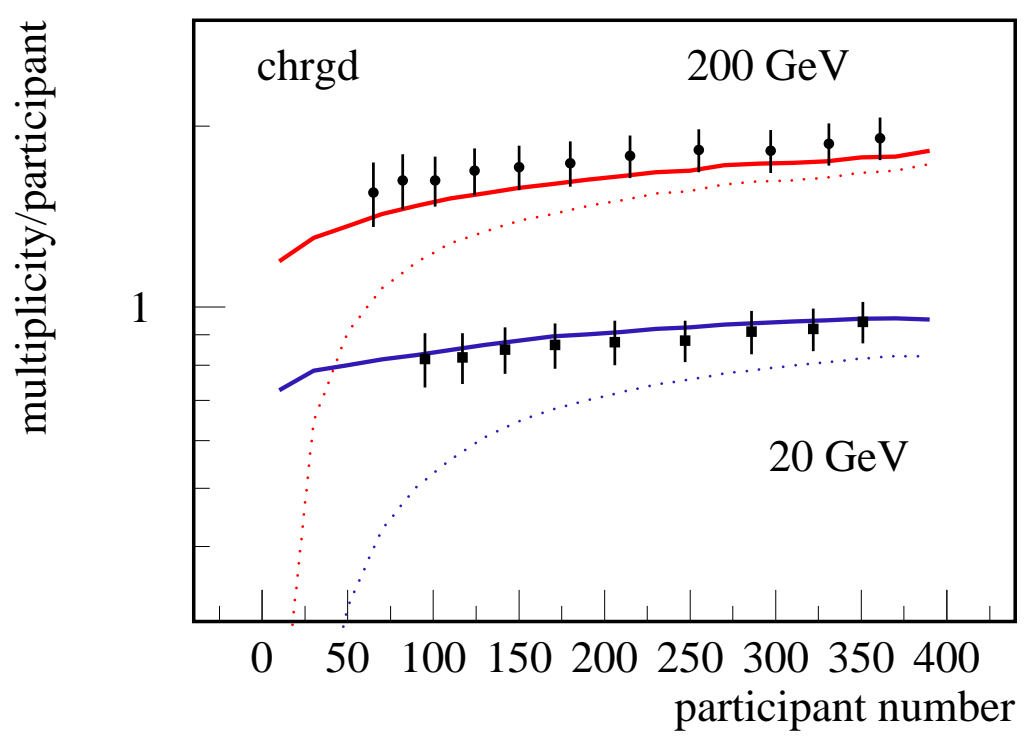

Figure 2: Rapidity density dn/dy of charged particles per participant as a function of the number of participants for $\mathrm{AuAu}$ at $200 \mathrm{GeV}$ and $20 \mathrm{GeV}$. We show data (points) [2] together with the full calculation (core + corona, full line) and just the core part (dashed).

whole procedure perfectly conserves energy, momentum, and flavors (microcanonical procedure).

In fig. 2, we compare the centrality dependence of the particle yields in AuAu collisions at $20 \mathrm{AGeV}$ and $200 \mathrm{AGeV}(\mathrm{cms})$. We show the data [2] together with the full calculation, but also indicating the core contribution. The complete calculation follows quite closely the data. Whereas central collisions are clearly core dominated, the core contributes less and less with decreasing centrality. We also observe that the corona contribution is more important at $20 \mathrm{GeV}$ than at 200 $\mathrm{GeV}$.

In fig. 3, we plot the elliptic flow of charged particles in AuAu collisions at 200, 62, and 20 $\mathrm{GeV}$, as a function of $\eta-y_{\text {proj }}$, where $\eta$ is the pseudo-rapidity and $y_{\text {proj }}$ the projectile rapidity. We show the data [3] compared to the full calculation and just the core part. Both data and simulations show what is sometimes referred to as "limiting fragmentation": for $\eta-y$ proj $\rightarrow 0$ the results for the three energies coincide, the physics in this area seems to be energy independent.

We discussed a new feature of the EPOS approach: the separation of the the collision zone in high energy nuclear collisions into a central part ("core") with expected high energy densities, and a peripheral part ("corona") with smaller energy densities more like in pp or pA collisions. The core part is parameterized in a simple fashion. We presented some results for particle production in heavy ion collisions between 20 and $200 \mathrm{GeV}$. In seems that in particular the centrality dependence of particle yields is strongly affected by the core-corona separation. 


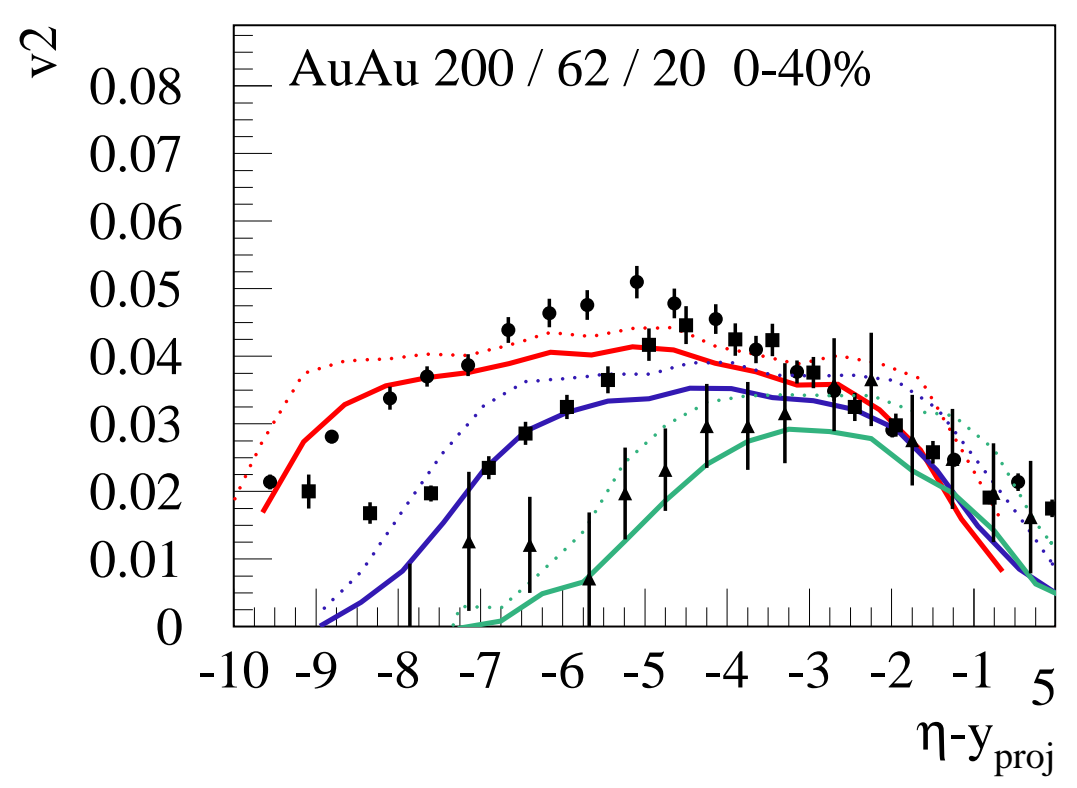

Figure 3: Elliptic fbw of charged particles in AuAu collisions at 200, 62, and $20 \mathrm{GeV}$, as a function of $\eta-y_{\text {proj }}$, where $\eta$ is the pseudo-rapidity and $y_{\text {proj }}$ the projectile rapidity. We show the data [3] (symbols) compared to the full calculation (full lines) and just the core part (dotted lines).

\section{References}

[1] K. Werner, Fuming Liu, Tanguy Pierog, J. Phys. G: Nucl. Part. Phys. 31 (2005) S985-S988.

[2] PHOBOS Collaboration, B.B.Back et al, Phys.Rev. C70 (2004) 021902

[3] PHOBOS Collaboration, B.B.Back, et al, Phys.Rev.Lett. 94 (2005) 122303 